\title{
An Improved Parameter Value Optimization Technique for the Reflectionless Transmission-Line Model of the Cochlea
}

\author{
Takemori Orima and Yoshihiko Horio \\ Research Institute of Electrical Communication, Tohoku University \\ 2-1-1 Katahira, Aoba-ku, Sendai, Miyagi, 980-8577, Japan \\ E-mail: takemori.orima.q2@dc.tohoku.ac.jp
}

\begin{abstract}
The passive reflectionless transmission-line model can reproduce physiological cochlear properties. We have applied an optimization technique to obtain good parameter values that give a desired cochlear property. However, we found that, in some case, it is difficult to find the sub-optimal parameter values because of complex dependencies among parameters. In this paper, we explicitly formulate some of the cochlear properties in the reflectionless transmission-line model, and improve the optimization technique using these formulae.
\end{abstract}

Keywords: cochlea, passive model, reflectionless transmission-line model, optimization technique.

\section{Introduction}

The physiological transmission-line model of the cochlea can reproduce the mechanical vibration of the basilar membrane well. To approximate individual human cochlear characteristics to experimental data, it is necessary to tune parameter values of the model. However, because of the large number of parameters, it is difficult to determine these values.

The passive reflectionless transmission-line model ${ }^{1}$ (passive model) has been proposed to alleviate this problem. Although this model has less number of parameters, it can reproduce the characteristics of the cochlea well. Therefore, we proposed a design technique for this model using parameter optimization ${ }^{2}$. However, this method requires a long calculation time to design.

In this paper, we explicitly give approximate formulae for some key characteristics of the passive model to accelerate the calculation. In addition, an optimization method is applied in the design technique with proposed formulae. We compare three optimization methods, and chose the best one for this design technique. Finally, we demonstrate some design examples to confirm the validity and efficiency of the proposed method.

\section{The passive reflectionless transmission-line model}

A minute part between $x$ and $x+\mathrm{d} x$ of the passive reflectionless transmission-line model ${ }^{1}$ is shown in Fig. 1 , where $x$ is a distance from the input terminal. The parallel impedance $Z_{p}(x, \omega)$ in Fig. 1 is given by

$$
Z_{p}(x, \omega)=j \omega L_{p}(x)+R_{p}(x)+\frac{1}{j \omega C_{p}(x)},
$$

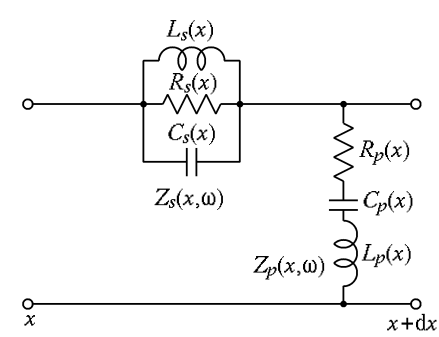

Fig. 1. A minute part between $x$ and $x+\mathrm{d} x$ of the passive reflectionless transmission-line model ${ }^{1}$. 
where $\omega$ is the angular frequency of the input signal.

Assuming that the value of each of the circuit elements in $Z_{p}(x, \omega)$ changes exponentially with distance, they can be given as

$$
R_{p}(x)=R_{0} e^{-a x}, L_{p}(x)=L_{0} e^{a x}, C_{p}(x)=C_{0} e^{a x},
$$

where $R_{0}, L_{0}$, and $C_{0}$ are values at $x=0$, and $a$ is a constant.

In Fig. 1, we further assume that the characteristic impedance $Z_{0}(x, \omega)$ is independent of the distance, so that

$$
Z_{0}(x, \omega)=r,
$$

where $r$ is a constant. As a result, the series impedance $Z_{s}(x, \omega)$ in Fig. 1 can be defined as

$$
Z_{s}(x, \omega)=\frac{r^{2}}{Z_{p}(x, \omega)} .
$$

On the other hand, the resonance angular frequency $\omega_{\text {res }}(x)$ is given by

$$
\omega_{r e s}(x)=\frac{1}{\sqrt{L_{0} C_{0}}} e^{-a x} .
$$

Taking the integral of the propagation constant with respect to $x$ yields

$$
\begin{gathered}
\Gamma(x, \omega)=\int_{0}^{x} \frac{r}{Z_{p}(x, \omega)} d x=\frac{j r}{2 a \sqrt{L_{0} / C_{0}+j \omega R_{0} L_{0}}} \\
\times\left\{\ln \left(\frac{\sqrt{1+j \omega R_{0} C_{0}}+\omega \sqrt{L_{0} C_{0}} e^{a x}}{\sqrt{1+j \omega R_{0} C_{0}}-\omega \sqrt{L_{0} C_{0}} e^{a x}}\right)\right. \\
\left.-\ln \left(\frac{\sqrt{1+j \omega R_{0} C_{0}}+\omega \sqrt{L_{0} C_{0}}}{\sqrt{1+j \omega R_{0} C_{0}}-\omega \sqrt{L_{0} C_{0}}}\right)\right\} .
\end{gathered}
$$

Finally, the transfer function is given by

$$
F(x, \omega)=\frac{1}{Z_{p}(x, \omega)} \exp (-\Gamma(x, \omega)) .
$$

\section{Explicit formulation of key characteristics of the passive model}

We explicitly formulate some of the key characteristics of the passive model, the center angular frequency $\omega_{c}(x)$, the maximum gain $g_{\max }(x)$, and the quality factor $Q(x)$ shown in Fig. 2 as follows:

$$
\begin{gathered}
\omega_{c}(x)=\mu_{c}(x) \omega_{r e s}(x), \\
g_{\text {max }}(x)=\mid F\left(x, \omega_{c}(x)\right),
\end{gathered}
$$

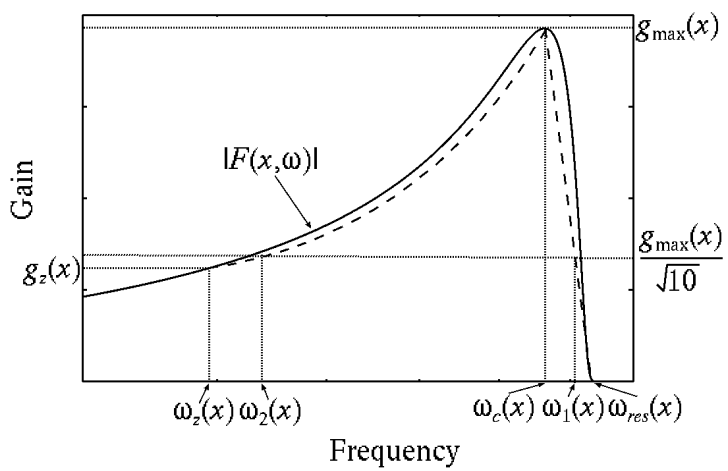

Fig. 2. The solid line: the frequency vs. gain characteristics of the original passive model obtained from Eq. (7). The dashed line: the approximation with Eqs. (13) and (14).

$$
Q(x)=\frac{\omega_{c}(x)}{\omega_{1}(x)-\omega_{2}(x)},
$$

where $\omega_{1}(x)$ and $\omega_{2}(x)$ are the angular frequencies at $g_{\max }(x) / \sqrt{10}$ given by

$$
\begin{gathered}
\omega_{1}(x)=\frac{1}{\sqrt{10}} \omega_{c}(x)+\left(1-\frac{1}{\sqrt{10}}\right) \omega_{\text {res }}(x), \\
\omega_{2}(x)=\frac{1}{A(x)} \ln \left(\frac{1}{B(x)} \cdot \frac{g_{\text {max }}(x)}{\sqrt{10}}\right),
\end{gathered}
$$

where

$$
\begin{gathered}
A(x)=\exp \left\{\frac{\omega_{c}(x) \ln g_{z}(x)-\omega_{z}(x) \ln g_{\max }(x)}{\omega_{c}(x)-\omega_{z}(x)}\right\}, \\
B(x)=\frac{\ln g_{\max }(x)-\ln g_{z}(x)}{\omega_{c}(x)-\omega_{z}(x)}, \\
g_{z}(x)=\mid F\left(x, \omega_{z}(x)\right), \\
\omega_{z}(x)=\mu_{z}(x) \omega_{r e s}(x), \\
\mu_{z}(x)=\sqrt{\frac{-v_{z}(x)-\sqrt{v_{z}(x)^{2}-4}}{2},} \\
v_{z}(x)=\frac{C_{0}}{L_{0}}\left\{\left(R_{0} e^{-a x}\right)^{2}-\left(\frac{\sqrt{10}}{g_{\max }(x)}\right)^{2}\right\}-2 .
\end{gathered}
$$

In addition, $\mu_{c}(x)$ is given by

$$
\mu_{c}(x)=\sqrt{\frac{-v_{c}(x)+\sqrt{v_{c}(x)^{2}+4}}{2}},
$$

where 


$$
v_{c}(x)=\frac{C_{0}}{L_{0}}\left(\frac{r}{a}-R_{0} e^{-a x}\right) R_{0} e^{-a x} .
$$

Comparisons between the original characteristics calculated numerically from Eq. (7) and approximated values from the above formulae are shown in Figs. 3 to 5 , where $a=0.288, R_{0}=1.5, L_{0}=2.385 \times 10^{-7}, C_{0}=$ $2.132 \times 10^{-7}$, and $r=5$. Equation (7) took about 0.26 sec. for calculation, in contrast, the formulae about 2.5 $\mu$ sec. From these results, we can confirm validities and efficiency of the proposed formulae.

\section{Design method using parameter optimization}

We design the passive model using the proposed formulae to reproduce the human cochlear characteristics. We employ an optimization technique to obtain the model parameters.

First, we define the object functions as follows:

$$
\begin{gathered}
E_{1}(x)=\left|\frac{\omega_{c}(x)-c f(x)}{c f(x)}\right|, \\
E_{2}(x)=\left|\frac{g_{\text {max }}(x)-m g(x)}{m g(x)}\right|, \\
E_{3}(x)=\left|\frac{Q(x)-q(x)}{q(x)}\right|,
\end{gathered}
$$

where $c f(x), m g(x)$, and $q(x)$ are the target values for the center frequency, maximum gain, and quality factor at a distance $x$, respectively. Then, we optimize the weighted sum of these object functions $E(x)$.

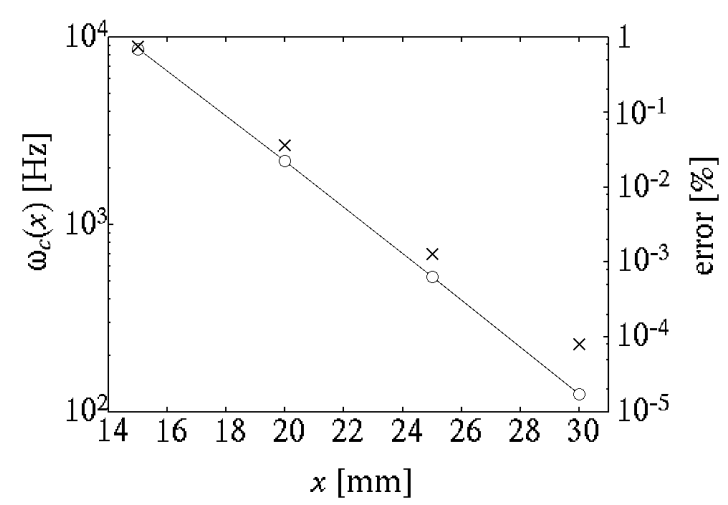

Fig. 3. The original (circles) and approximated (solid line) center frequencies $\omega_{c}(x)$ according to the distance $x$ obtained from Eq. (7) and Eq. (8), respectively. The crosses: the percent errors between them.

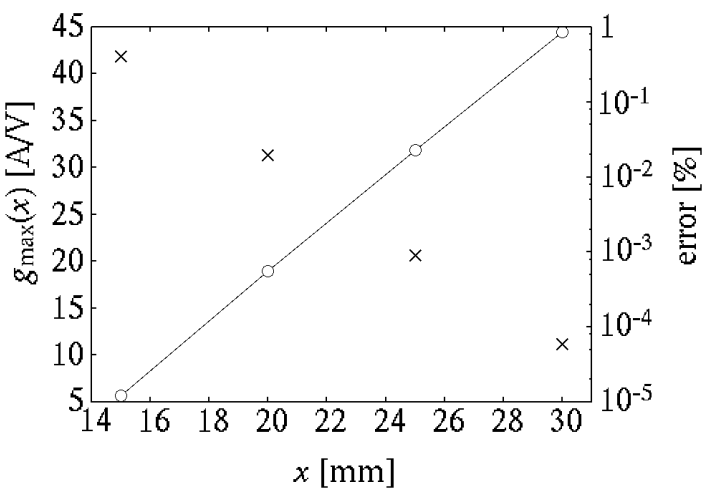

Fig. 4. The original (circles) and approximated (solid line) maximum gains $g_{\max }(x)$ according to the distance $x$ obtained from Eq. (7) and Eq. (9), respectively. The crosses: the percent errors between them.

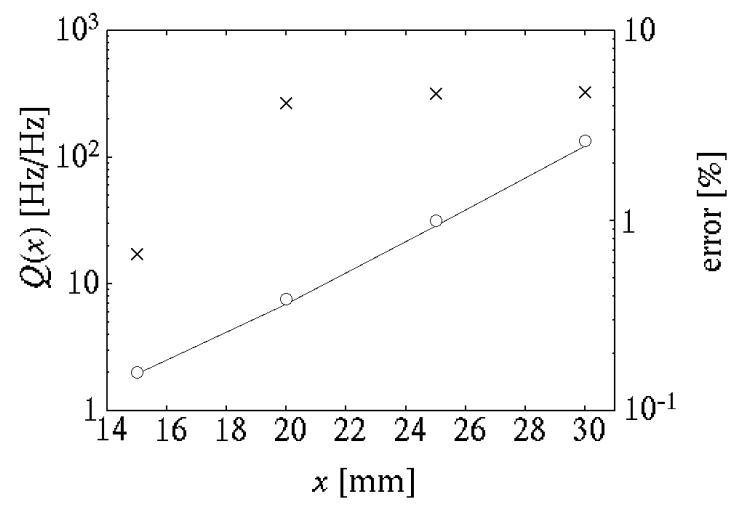

Fig. 5. The original (circles) and approximated (solid line) quality factors $Q(x)$ according to the distance $x$ obtained from Eq. (7) and Eq. (10), respectively. The crosses: the percent errors between them.

$$
E(x)=\sum_{i=1}^{3} w_{i} E_{i}(x) \text { and } \sum_{\mathrm{i}=1}^{3} w_{i}=1,
$$

where $w_{i} \geq 0$ is a weight. In the following section, we design the passive model with the target parameter values shown in Table 1, where $c f(x), m g(x)$, and $q(x)$ were estimated based on the physiological experiments $^{3,4}$.

\subsection{Optimization techniques}

We compare the downhill simplex (DHS), the genetic algorithm (GA), and the particle swarm

Table 1. The target design specifications.

\begin{tabular}{|c|c|c|c|}
\hline$x$ & $15 \mathrm{~mm}$ & $20 \mathrm{~mm}$ & $25 \mathrm{~mm}$ \\
\hline$c f(x)[\mathrm{Hz}]$ & 2500 & 1200 & 500 \\
\hline$m g(x)[\mathrm{A} / \mathrm{V}]$ & 9.4 & 9.4 & 6.1 \\
\hline$q(x)[\mathrm{Hz} / \mathrm{Hz}]$ & 3.6 & 2.6 & 1.8 \\
\hline
\end{tabular}


optimization (PSO), using the design target at $x=25$ $\mathrm{mm}$ in Table 1 . The conditions of each optimization methods are summarized in Table 2. In the table, $N$ is the number of simplexes, individuals, and particles in DHS, GA, and PSO, respectively. Note that, in the case of PSO, one iteration is counted each time as the global best is updated.

The results of the comparisons are shown in Table 3. In the table, DHS gives the smallest optimization function value. Therefore, we use DHS as the optimization method in our design.

\subsection{Design examples}

We designed the passive model specified in Table 1 using DHS. The results are shown in Table 4 and Fig. 6. From Table 4, we confirm that the designed values are almost the same as the target ones, although some errors remain due to approximation errors in the proposed formulae, in particular, Eq. (10).

\section{Conclusion}

We have explicitly formulated some key characteristics of the passive reflectionless transmission-line model of the cochlea to alleviate the complex calculation loads. These expressions efficiently reproduced the original characteristics.

In addition, we defined the object functions for parameter optimizations. We compared DHS, GA, and PSO as the optimization methods. As a result, we found that DHS is the best method.

We finally designed three cochlear characteristics at different distances successfully using the proposed formulae with DHS.

As a future problem, we will improve the formulae for $Q(x)$ for more precise designs.

Table 2. The simulation conditions.

\begin{tabular}{|c|c|c|c|}
\hline & DHS & GA & PSO \\
\hline$N$ & 30 & 30 & 30 \\
\hline Trials & 30 & 30 & 30 \\
\hline Iterations & 5000 & 5000 & 5000 \\
\hline
\end{tabular}

\section{Acknowledgments}

This work was supported, in part, by JSPS KAKENHI Grant Number 16K00340, and the Cooperative Research Program of the Research Institute of Electrical Communication, Tohoku University. The authors are grateful to Emeritus Professor Tohru Kohda of Kyushu University for his valuable discussions.
Table 3. The obtained parameters and object function values with DHS, GA, and PSO for $x=25 \mathrm{~mm}$.

\begin{tabular}{|c|c|c|c|}
\hline & DHS & GA & PSO \\
\hline \hline$a$ & 0.1949 & 0.2299 & 0.2141 \\
\hline$R_{p}$ & $2.315 \times 10^{-3}$ & $2.596 \times 10^{-3}$ & $2.816 \times 10^{-3}$ \\
\hline$L_{p}$ & $4.963 \times 10^{-9}$ & $8.227 \times 10^{-10}$ & $2.122 \times 10^{-9}$ \\
\hline$C_{p}$ & $5.875 \times 10^{-8}$ & $1.068 \times 10^{-8}$ & $2.071 \times 10^{-8}$ \\
\hline$r$ & 2.5 & 2.5 & 2.5 \\
\hline \hline$E$ (Eq. (21)) & $1.47 \times 10^{-6}$ & $1.34 \times 10^{-4}$ & $6.38 \times 10^{-2}$ \\
\hline
\end{tabular}

Table 4. The obtained center frequencies $\omega_{c}(x)$, maximum gains $g_{\max }(x)$, and quality factors $Q(x)$ by the proposed design technique with DHS.

\begin{tabular}{|c|c|c|c|}
\hline$x$ & $15 \mathrm{~mm}$ & $20 \mathrm{~mm}$ & $25 \mathrm{~mm}$ \\
\hline$\omega_{c}(x)[\mathrm{Hz}]$ & 2496 & 1196 & 495 \\
\hline$g_{\max }(x)[\mathrm{A} / \mathrm{V}]$ & 9.408 & 9.417 & 6.133 \\
\hline$Q(x)[\mathrm{Hz} / \mathrm{Hz}]$ & 3.858 & 2.733 & 1.834 \\
\hline
\end{tabular}

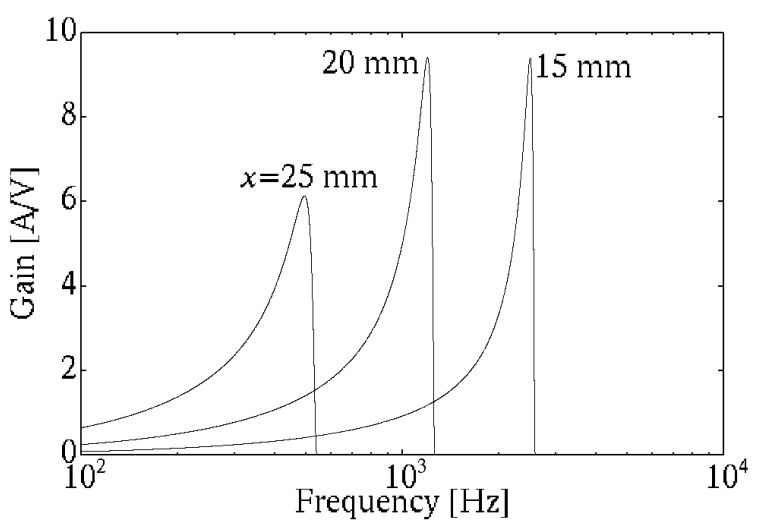

Fig. 6. The obtained frequency vs. gain characteristics at $x=$ $15 \mathrm{~mm}, 20 \mathrm{~mm}$, and $25 \mathrm{~mm}$ through the proposed optimization technique using downhill simplex method.

\section{References}

1. T. Kohda, T. Une, and K. Aihara, "An active, reflectionless transmission-line model of the cochlea: Revisited," in AIP Conf. Proc., vol. 1403, no. 1, pp. 578583, 2011; DOI:10.1063/1.3658152.

2. T. Orima, Y. Horio, and T. Kohda, "A parameter value optimization technique for a reflectionless transmissionline model of the cochlea," in Proc. of Int. Symp. on NOLTA2016, pp. 503-506, 2015.

3. G. V. Bekesy, "Experiments in Hearing," McGraw-Hill Book Co., New York, 1960.

4. M. A. Ruggero and A. N. Temchin, "Unexceptional sharpness of frequency tuning in the human cochlea," PNAS, vol. 102, no. 51, pp. 18614-18619, 2005; DOI:10. 1073/PNAS.0509323102. 